\title{
BMJ Open Study protocol on advance care planning in multiple sclerosis (ConCure-SM): intervention construction and multicentre feasibility trial
}

\author{
Ludovica De Panfilis, ${ }^{1}$ Simone Veronese, ${ }^{2}$ Michela Bruzzone, ${ }^{3}$ Marta Cascioli, ${ }^{4}$ \\ Alberto Gajofatto, ${ }^{5,6}$ Maria Grazia Grasso, ${ }^{7}$ Paola Kruger, ${ }^{8}$ Alessandra Lugaresi, ${ }^{9,10}$ \\ Leigh Manson, ${ }^{11}$ Sara Montepietra, ${ }^{12}$ Francesco Patti, ${ }^{13}$ Eugenio Pucci, ${ }^{14}$ \\ Claudio Solaro, ${ }^{15}$ Andrea Giordano, ${ }^{16}$ Alessandra Solari (i) ${ }^{16}$
}

To cite: De Panfilis L, Veronese S, Bruzzone $\mathrm{M}$, et al. Study protocol on advance care planning in multiple sclerosis (ConCure-SM): intervention construction and multicentre feasibility trial. BMJ Open 2021;11:e052012. doi:10.1136/ bmjopen-2021-052012

- Prepublication history and additional supplemental material for this paper are available online. To view these files, please visit the journal online (http://dx.doi.org/10.1136/ bmjopen-2021-052012).

Received 02 April 2021 Accepted 02 August 2021

A) Check for updates

(c) Author(s) (or their employer(s)) 2021. Re-use permitted under CC BY-NC. No commercial re-use. See rights and permissions. Published by BMJ.

For numbered affiliations see end of article.

\section{Correspondence to}

Dr Alessandra Solari; alessandra.solari@istitutobesta.it

\section{ABSTRACT}

Introduction Multiple sclerosis (MS) is the most common cause of progressive neurological disability in young adults. The use of advance care planning (ACP) for people with progressive MS (pwPMS) remains limited. The ConCure-SM project aims to assess the effectiveness of a structured ACP intervention for pwPMS. The intervention consists of a training programme on ACP for healthcare professionals caring for pwPMS, and a booklet to be used during the ACP conversation. Herein, we describe the first two project phases.

Methods In phase 1 we translated and adapted, to the Italian legislation and MS context, the ACP booklet of the National ACP Programme for New Zealand. Acceptability, comprehensibility and usefulness of the booklet were assessed via 13 personal cognitive interviews with pwPMS and significant others (SOs), and one health professional focus group. Based on these findings, we will revise the booklet. In phase 2 we will conduct a single-arm pilot/ feasibility trial with nested qualitative study. Participants will be 40 pwPMS, their S0s, health professionals from six MS and rehabilitation centres in Italy. In the 6 months following the ACP conversation, we will assess completion of an advance care plan document (primary outcome), as well as safety of the intervention. Secondary outcomes will be a range of measures to capture the full process of ACP; patient-carer congruence in treatment preferences; quality of patient-clinician communication and caregiver burden. A qualitative process evaluation will help understand the factors likely to influence future implementation and scalability of the intervention.

Ethics and dissemination The project is coleaded by a neurologist and a bioethicist. Phase 1 has received ethical approvals from each participating centre, while phase 2 will be submitted to the centres in May 2021. Findings from both phases will be disseminated widely through peer-reviewed publications, conferences and workshops. Trial registration number ISRCTN48527663; Pre-results.

\section{INTRODUCTION}

With a lifetime risk of 1 in 400, multiple sclerosis (MS) is the most common cause of progressive neurological disability in young

\section{Strengths and limitations of this study}

- A strength of the study is the use of a mixedmethods approach.

- The intervention is coproduced with users.

- Study results will be key to inform the feasibility of a full-scale trial, and its design.

- A limitation is that the pilot trial is a non-randomised study.

- Long-term outcomes (chiefly concordance between preferred and received end-of-life care) are not included.

adults. Approximately 2.3 million people worldwide have MS, with Canada, the USA and some European countries, including Italy, having the highest prevalence rates. ${ }^{1}$ Around $15 \%$ of people with MS have a primary progressive course at diagnosis, and a further $35 \%$ develop secondary progressive disease after 15 years. ${ }^{2}$ A mean reduction in life expectancy by 7-14 years has been reported in people with MS, with improved figures over the last two decades. ${ }^{3-5}$

Few treatment options are currently available to delay or prevent further clinical worsening of people with primary or secondary progressive MS (pwPMS). They may live for many years experiencing a wide range of symptoms, impairments (including cognitive impairment which affects $40 \%-70 \%$ of sufferers $^{6}$ ) and comorbidities. ${ }^{5-10}$

Advance care planning (ACP) is a process that 'enables individuals who have decisional capacity to identify their values, to reflect upon the meanings and consequences of serious illness scenarios, to define goals and preferences for future medical treatment and care, and to discuss these with family and healthcare professionals (HPs)'.11 
Consistently with the Shared Decision Making model, ${ }^{12-14}$ ACP involves both the patient and his/her HPs. Together, they make informed decisions about the patient's (future) care. Also, the family can be involved in the process, if the patient wishes. ACP differs from general medical decision-making in that it is based on an anticipated deterioration in the health of a patient. It includes a focus on the person's wishes and preferences for the time when they lose decisional capacity. In fact, it aims to align evidence-based practice and person-centred care ${ }^{15}$ using a bioethical focus to identify the patient's values, preferences and desires. The planning process helps the patient to identify his/her personal values and goals, understand his/her health status, and the treatment and healthcare options available. Finally, ACP encourages discussion around end-of-life (EOL) care (a subject that is generally not considered part of healthcare planning, and one that can be avoided by both patients and HPs). It is up to the patient to determine the occurrence and content of any ACP discussion: if the patient does not wish to engage in conversations about his/her future care, this preference should be respected. The ACP process may result in the patient choosing to write an advance care plan document and to appoint a trustee (or else).

On 22 December 2017, the Italian Parliament approved the first law on EOL: 'Provisions for informed consent and advance directives' (L. 219/2017; http://www.trovanorme. salute.gov.it/norme/dettaglioAtto?id=62663) . This law regulates advance directives (AD; Article 4) and ACP (Article 5), and a number of rights citizens have regarding healthcare issues, including the right to: be fully informed about one's health status and to give consent (or dissent) to treatment; withhold consent to lifesaving treatments; be assisted until death. Moreover, the law states that the physician has a duty to respect the patient's wishes. In a recent Italian survey, $88 \%$ (1752/2000) of citizens considered the Law 219/2017 as quite or very important, and $76 \%$ had a positive attitude towards making/registering $\mathrm{AD}$ or $\mathrm{ACP}{ }^{16}$ Importantly, this Law triggers HPs and healthcare authorities in promoting educational programmes on the topic, as well as programmes to implement ACP in daily clinical practice.

To optimise the alignment between patient preferences and values and the care they receive. HPs should integrate best ACP practices in the care of pwPMS. A recent guideline on palliative care in MS found no evidence of the effects of ACP in pwPMS. ${ }^{17}$ However, there is some evidence from non-neurological progressive and lifethreatening illnesses that ACP decreases the use of lifesustaining treatment, increases hospice/palliative care, reduces hospitalisations and increases alignment with patients' EOL wishes. ${ }^{18}$ Furthermore, there is evidence that patients with MS and caregivers often would like to discuss the issues of death and dying and HPs should acknowledge and encourage these discussions. ${ }^{19}{ }^{20}$ However, often HPs leave EOL discussions until the later stages of progression in $\mathrm{MS},{ }^{21}$ and caregivers may be left having to take difficult decisions. ${ }^{22} \mathrm{~A}$ realist review identified two main barriers for ACP discussions taking place: the long and uncertain MS trajectory, with periods of stability punctuated by crisis; and lack of ACP communication skills and confidence of HPs. ${ }^{23}$

ConCure-SM is a project aimed to set up and evaluate the efficacy of an ACP intervention for pwPMS in Italy. The Shared Decision Making model described earlier is the theoretical framework of the project. ${ }^{12-14}$ The Medical Research Council framework for developing and evaluating complex interventions is the methodological framework of the project. The framework has a phased approach, from a preclinical research phase to a final phase in which the intervention is introduced into the health service, leading to a theory-driven intervention: a 'bottom-up' development which guarantees to enter a phase III trial with an appropriate theory and pilot work. ${ }^{24}$ Furthermore, both quantitative and qualitative methods are integrated within the framework, in order to better appraise the effects of the (complex) intervention both as a whole and on its components.

Our study hypotheses are that the intervention will produce: higher completion of an advance care plan document; increased congruence in treatment preferences between pwPMS and their carers; increased quality communication about EOL care.

\section{METHODS AND ANALYSIS}

The study protocol (FISM 2020/R-Multi/024; V.1.0; 15 March 2021) was designed following the Standard Protocol Items: Recommendations for Interventional Trials (SPIRIT) guidelines (online supplemental appendix 1$)^{25}$ and the SPIRIT-PRO Extension. ${ }^{26}$ The pilot/feasibility study follows the Consolidated Standards of Reporting Trials (CONSORT) guidance for trials on social and psychological interventions (CONSORT-SPI 2018) ${ }^{27}$ It was registered on the ISRCTN registry on 30 March 2021. Qualitative data will be reported following the Consolidated criteria for Reporting Qualitative research checklist. ${ }^{28}$

Figure 1 outlines the two project phases and inscribed actions. The red dot identifies the current advancement status.

\section{Phase 1}

The first project phase involves production of the ACP booklet (figure 1).

\section{Provisional booklet}

Early in 2020, an interdisciplinary panel translated into Italian and adapted to the MS context and to the Italian legislation, the ACP booklet of the Health Quality \& Safety Commission's New Zealand National ACP Programme (https://www.myacp.org.nz). The panel was made of five neurologists, one palliative care physician, one palliative care nurse, one psychologist, one bioethicist, one expert patient, one representative of the Italian MS Society and 


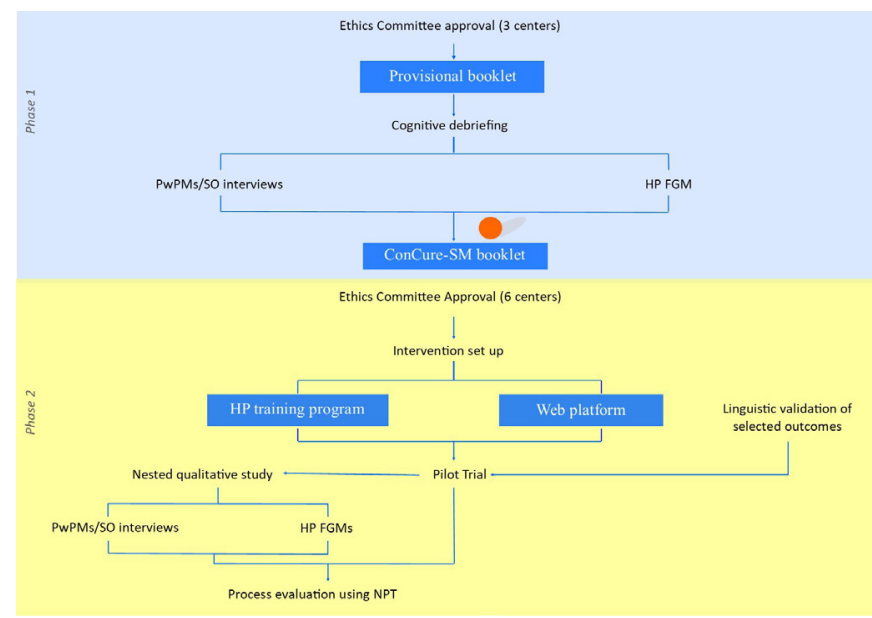

Figure 1 Flow chart of the ConCure-SM project. The red dot identifies the advancement status at the time of manuscript submission. FGM, focus group meeting; HP, health professional; MS, multiple sclerosis; NPT, normalisation process theory; pwPMS, people with progressive MS; SO, significant other.

the author of the original booklet. The resulting booklet in its provisional version (online supplemental appendix 2) consists of an introduction, a 'guidance' (the odd pages in most instances) and the advance care plan document (the even pages) to be completed electronically or manually by the pwPMS together with his/her referring physician. A significant other ( $\mathrm{SO}$ ), such as a family member, can participate in the process if requested by the pwPMS. The introduction explains the concepts of ACP and AD according to the Italian Law 219/2017, and describes why ACP is important in MS. Ten sections follow: 'My Advance Care Plan'; 'What matters to me'; 'What worries me'; 'Why I'm making an Advance Care Plan'; 'How I make decisions'; 'If I were no longer able to make decisions: my trustee'; 'Thinking about my EOL'; 'My treatment and care choices'; 'Signatures'; 'Acronyms'. If the advance care plan document is completed, the pwPMS (and, when applicable, the pwPMS trustee) sign on page 29; the document is then scanned and stored, together with the completed booklet, in the (electronic) medical record.

\section{Users' assessment and revision}

Between September and November 2020, the acceptability (contents, format, envisaged administration procedure), comprehensibility and usefulness of the provisional booklet were assessed by conducting 13 personal cognitive interviews with pwPMS, pwPMS' SOs and a focus group meeting (FGM) with HPs. Due to the COVID-19 pandemic, all the interviews and the FGM were held using digital platforms. Results of the qualitative (thematic) analysis and the revision of the booklet are underway.

\section{Phase 2}

The second project phase will be dedicated to the conduction of the multi-centre, pilot and feasibility single-arm trial with a nested qualitative study. This phase (to be accomplished from May 2021 to November 2022) has three inscribed actions: intervention set up; pilot trial and qualitative study (figure 1).

\section{Intervention set up}

\section{Training programme}

The goal of this intervention is to prime HPs to discuss goals of care and ACP. To achieve this, HPs will attend a training programme (called Train-ConCure-SM) that will be Continuing Medical Education accredited, residential, and last one-and-half days (12 hours). The programme aims to: improve the HP knowledge, competencies and skills in ACP based on up-to-date scientific evidence; support and guide HPs in the ACP embedment in clinical practice; improve the communication between HPs and patient promoting an effective patient-practitioner partnership in decision-making.

The training will be interactive in style. Its residential nature and the use of role-playing exercises aim at supporting group discussion and the exchange of experiences between participants.

It will consist of the following: one 2.5-hour theoretical session on the clinical, ethical and statutory principles of Shared Decision Making and ACP; two 4-hour empirical sessions (one on each day) on conducting ACP conversations in various clinical scenarios using the ConCure-SM booklet through guided role play exercises; two 45-min self-evaluation sessions (at the beginning and at the end of the training programme).

Trainees will be physicians and other HPs from the six enrolling centres. The Italian Law 219/2017 prescribes that ACP involves the patient, his/her referring physician and (when applicable) the trustee. We decided to train HPs other than physicians in order to promote ACP knowledge within the caring team. Each centre will provide 1-3 physicians, plus one HP from the following: MS nurse, therapist, psychologist or social worker. Thus, there will be 12-24 participants overall (2-4 from each centre). Trainers will be a panel of neurologists, psychologists, a palliative care physician, a palliative care nurse and a bioethicist. All have consolidated experience in leading training courses and workshops on patientclinician communication and Shared Decision Making, and four on ACP and EOL conversations. These four researchers will support physicians at the centres for issues concerning the conduction of the ACP conversation during the pilot trial.

\section{Web platform}

As part of the intervention set up action, a web-based trial platform will be created containing the pseudoanonymised trial case record form (eCRF) and the outcome measures. The platform will be ID/password protected, with dedicated accesses based on the stakeholder (pwPMS, SO, HPs, centre principal investigator (PI), interviewer, data manager) and operation (completion, consultation). 


\section{Linguistic validation of measures}

Two outcome measures not available in Italian will be translated and cross-culturally adapted, following accepted guidelines ${ }^{29}$ : : the 4-item ACP Engagement questionnaire (4-item ACP-E) ${ }^{31}$ and the Quality of Communication Questionnaire (QOC). ${ }^{32}$ The main steps in this process are the following:

- Forward translation: two qualified translators, both living in Italy, will produce two independent translations. A panel consisting of the translators, two MS HPs and two lay persons will review the forward translations and a consensus version will be produced.

- Backward translation: the consensus translation generated in step 1 will be independently translated back into the source language by a third qualified translator, living in the target country. The backward translation will be produced without access to the original version and without consulting the other translators.

- Translation refinement: in a meeting between those participating in step 1 and the backward translator, the backward translation will be compared with the original, and further refinements to the Italian version will be made. Differences will be resolved by discussion.

- Each translated questionnaire will be proofread, and then administered to/debriefed with 5-10 patients.

\section{Pilot and feasibility trial}

The six centres involved in the pilot trial are located in northern (four centres), central and southern Italy (one centre each). Two of the centres are rehabilitation hospitals (one of which a research hospital), three are MS centres (two university hospitals, one research hospital) and one is a rehabilitation and MS centre from a research hospital. Recruitment will be competitive, with no prespecified minimum number of enrolled subjects per centre. The maximum number of enrolled subjects per centre is 12 .

There will be a baseline assessment (T0), an ACP conversation taking place within 1 month from the baseline assessment, and a follow-up assessment within 1 week of the ACP conversation (T1) and 6 months (T2) thereafter. The baseline and follow-up assessments will be performed via the web-based ConCure-SM platform. The physician will record on the platform subsequent ACP conversations that should occur during follow-up. Participants (pwPMS, SOs) will be free to withdraw from the study at any time, without giving reasons and with no risk of prejudicing future care. Study personnel will make every effort to obtain, and record, information about the drop out reasons.

The objectives of the pilot and feasibility trial are reported in box 1. trial procedures are summarised in figure 2 .

\section{Eligibility and screening}

PwPMS (inpatients or outpatients) will be included if they are: $\geq 18$ years of age; diagnosed with primary or

\section{Box 1 Objectives of the pilot trial}

- To determine how many people with progressive multiple sclerosis accept the invitation to participate in the study.

- To determine how many participants receive the intervention.

- To estimate recruitment and refusal rates, and 6-month follow-up rates.

- To estimate advance care planning (ACP) completion during the 6-month follow-up (primary study outcome).

- To estimate occurrence of serious adverse events and adverse events during the 6-month follow-up.

- To assess, qualitatively, the acceptability of the recruitment processes, assessments, intervention delivery and secondary outcome measures with key stakeholders.

- To measure changes in the secondary outcome measures.

- To explore the barriers and facilitators to implementing ACP in pwPMS, and the influence of the clinical setting.

- To inform the sample size estimation for a subsequent phase III trial, should this be feasible.

secondary PMS $^{33}$ one or more years before inclusion; able to communicate in Italian and gave written consent. In addition, one or more of the following conditions that would make ACP relevant must be present: expressed desire for ACP; questions about own future; thoughts about hastening death or medically assisted suicide; high risk for death within 2 years using the 'Surprise Question, ${ }^{34}$; high risk for development of severe cognitive compromise/dementia within 2 years; high risk for development of impairments preventing communication within 2 years; significant suffering (eg, uncontrolled physical symptoms, psychosocial or existential issues). PwPMS will be excluded if they have one or more of the following: severe cognitive compromise (MMSE <19) or impairments preventing communication; psychosis or other serious psychiatric conditions; advance care plan document completed.

PwPMS are recruited prospectively by the ACP-trained physicians involved in their care, when the potentially eligible pwPMS attends the centre for an outpatient visit or hospitalisation. PwPMS who show interest in participating receive full verbal and written information about the study purpose and procedures.

\section{Baseline assessment (TO)}

The ACP-trained physician makes an appointment with pwPMS who provided initial verbal consent to participate in the study, and checks all eligibility criteria. A written, signed informed consent is obtained, according to the Declaration of Helsinki and to the Good Clinical Practice (GCP) guidelines of the EU. The informed consent is kept on file by the study personnel, and is available for inspection by regulatory authorities or authorised persons.

Then, the physician gives the pwPMS the credentials to the trial platform, so that the pwPMS completes the baseline set of questionnaires/instruments (completion time around $40 \mathrm{~min}$ ). If the pwPMS has difficulties in using the trial platform, a telephone interview is scheduled within 
Eligible pwPMS:

Age $\geq 18$ years

- At least one out of seven conditions that would make ACP relevant

- Able to communicate in Italian

- Adequate cognitive and communicative ability to participate

- No serious psychiatric conditions

- No previous advance care plan document completed

Participant screening:

- Confirm eligibility

- Obtain name/contact of significant other (if applicable) and permission to contact

Baseline assessment (TO):

\begin{tabular}{|c|c|}
\hline PwPMS & $\begin{array}{l}\text { - HADS } \\
\text { - eCPS } \\
\text { - 4-item ACP Engagement } \\
\text { - MSQOL-29 }\end{array}$ \\
\hline Significant other & $\begin{array}{l}\text { - General data } \\
\text { - ZBI }\end{array}$ \\
\hline Physician & $\begin{array}{l}\text { - PwPMS general and clinical data (EDSS [Kurtzke 1983], } \\
\text { Barthel Index [Mahoney 1965]) } \\
\text { - Physician's general data }\end{array}$ \\
\hline
\end{tabular}

First ACP conversation: - OPTION scale (physician's competences)

Follow-up assessment (T1):

Follow-up assessment (T2):

\begin{aligned} PwPMS & $>$ HADS \\ & $>$ QOC \\ \cline { 2 - 2 } Significant other & $>\mathrm{ZBC} \\ & >\mathrm{QOC}-\mathrm{SO} \\$ Physician & $>$ QOC-DoC \\ PwPMS & $>$ HADS \\ & $>4$-item ACP Engagement \\ & $>$ MSQOL-29 \\ Significant other & $>$ ZBI \\ Physician & $>$ PWPMS clinical/ACP update \end{aligned}

Figure 2 Summary of trial procedures. ACP, advance care planning; ACP-E, ACP Engagement; eCPS, Control Preference Scale, electronic; EDSS, Expanded Disability Status Scale; HADS, Hospital Anxiety and Depression Scale; MSQOL-29, 29item Multiple Sclerosis Quality of Life; OPTION, Observing Patient Involvement in Shared Decision Making; QOC, Quality of Communication; SO, significant other; ZBI, Zarit Burden Interview. Kurtzke ${ }^{58}$; Mahoney and Barthel. ${ }^{59}$

a week with an independent, trained interviewer who will administer the questionnaires/instruments.

The ACP conversation is scheduled at the centre, within a month. It is the starting point of a process that is followed up during the study. However, for feasibility reasons and to adapt to participant needs, subsequent conversations are recorded, but not scheduled a priori. The pwPMS is invited to involve his/her SO (family member, relative or friend, who is next of kin or is key decision-maker as designated by the pwPMS and with whom the pwPMS shares his/her life). If the pwPMS agrees on involving his/her $\mathrm{SO}$, the $\mathrm{SO}$ is contacted by a study researcher to confirm eligibility, explain the study and obtain verbal consent. Consenting SOs receive credentials to access the trial platform and complete the baseline set of questionnaires (completion time about $15 \mathrm{~min}$ ). If the SO has difficulty 
in using the trial platform, a telephone interview is scheduled within a week with an independent, trained interviewer who will administer the questionnaires.

Finally, the physician completes the eCRF via the trial platform.

Each centre will collect information on the number of pwPMS and SOs approached, screened and eligible prior to enrolment, with reasons for non-enrolment.

\section{ACP conversation}

The conversation involves the pwPMS, the ACP-trained physician involved in his/her care and, when applicable, the SO. In addition, if the pwPMS agrees, the nonphysician ACP-trained HP at the centre will participate. The first conversation takes place in a dedicated room at the centre, and is audio-recorded. At MS centres and rehabilitation centres, physician time and space are at premium, particularly for outpatient care. For this reason, 1-hour slot is reserved for the conversation. In the case an SO participates it is envisaged that there will be a session closed to the SO, followed by an open session.

About 1 week before the scheduled ACP conversation, reminder emails (or telephone calls) are sent to pwPMS/ SOs. At the end of the ACP conversation, the physician invites the pwPMS (when applicable the SO) to complete the T1 follow-up assessment within 1 week. The physician completes the QOC-Doc immediately after the ACP conversation ends.

\section{Follow-up assessments (T1, T2)}

The pwPMS completes the questionnaires by 1 week (T1, assessment time of about $20 \mathrm{~min}$ ) and 6 months (T2, assessment time of about $30 \mathrm{~min}$ ) after the first ACP conversation using the trial platform. The SO completes the questionnaires/instruments ( $\mathrm{T} 1$, assessment time of about $20 \mathrm{~min}$ ) using the trial platform. In the event the pwPMS/SO have difficulties in using the trial platform, a telephone interview is scheduled with an independent, trained interviewer who will administer the questionnaires/instruments.

About 1 week before the T2 assessment, reminder emails (or telephone calls) are sent to pwPMS. The physician completes the questionnaire (T1, QOC-Doc) and the eCRF using the trial platform. $\mathrm{He} /$ she records on the platform the date, duration, participants and mode (face to face, teleconference or on the telephone) of subsequent ACP conversations that occur during follow-up.

\section{Outcome measures}

A range of measures will be collected to capture the full process of ACP and whether the ConCure-SM intervention has any effect on completion of an advance care plan document (primary outcome measure), congruence in treatment preferences between pwPMS and their carers, quality of patient-clinician communication and caregiver burden (table 1). In addition, since a study-related increase in emotional burden can not be excluded, serious adverse events (admission to psychiatric ward, suicide attempt, death) will be monitored by the independent Data and Safety Monitoring Committee (DSMC).

We will use the published Italian version of the following inventories: Control Preference Scale (CPS) ${ }^{35}$; Hospital Anxiety and Depression Scale (HADS) ${ }^{36}$; Observing Patient Involvement in Decision Making (OPTION) ${ }^{37}$; 29-item Multiple Sclerosis Quality of Life (MSQOL)-29 ${ }^{38}$; Zarit Burden Interview (ZBI). ${ }^{39}$ The 4 -item ACP-E and the QOC inventories will be translated/culturally adapted from the source language (see previously).

\section{ACP engagement}

The ACP process will be assessed using the 4-item ACP-E questionnaire. ${ }^{31}$ Originally developed and validated to measure the complex behaviour of ACP, the questionnaire is available in four versions (55-item, 34-item, 9-item, 4-item). In this study, we will use the 4-item version which focuses on the readiness behaviour change construct

\begin{tabular}{|c|c|c|c|c|c|}
\hline Scale name & Assessor & Construct & Author & Italian version & Timing \\
\hline 4-item ACP-E & Patient & ACP engagement & Sudore et $a^{31}$ & - & $\mathrm{T} 0 / \mathrm{T} 1 / \mathrm{T} 2$ \\
\hline HADS & Patient & Mood symptoms & $\begin{array}{l}\text { Zigmond and } \\
\text { Snaith }^{44}\end{array}$ & Costantini et $a l^{36}$ & $\mathrm{TO} / \mathrm{T} 1 / \mathrm{T} 2$ \\
\hline QOC & Patient & Communication quality (physician's skills) & Engelberg et $a l^{32}$ & - & $\mathrm{T} 1$ \\
\hline QOC-Doc & Physician & Communication quality (physician's skills) & - & - & $\mathrm{T} 1$ \\
\hline QOC-SO & SO & Communication quality (physician's skills) & - & - & $\mathrm{T} 1$ \\
\hline ZBI & SO & Caregiver burden & Hérbert et $a l^{46}$ & Chattat et al ${ }^{39}$ & $\mathrm{~T} 0 / \mathrm{T} 1 / \mathrm{T} 2$ \\
\hline
\end{tabular}

ACP-E, Advance Care Planning Engagement; eCPS, Control Preference Scale, electronic; HADS, Hospital Anxiety and Depression Scale; MSQOL-29, 29-item Multiple Sclerosis Quality of Life; OPTION, Observing Patient Involvement in Shared Decision Making; QOC, Quality of Communication; SO, significant other; ZBI, Zarit Burden Interview. 
within the quality of life ACP domain. Responses are on a 5-point Likert scale ((1) 'I have never thought about it'; (2) 'I have thought about it, but I am not ready to do it'; (3) 'I am thinking about doing it in the next 6 months'; (4) 'I am definitely planning to do it in the next 30 days'; (5) 'I have already done it'). ${ }^{31}$

\section{Role preferences}

The CPS is the most used instrument to assess patient preferences for involvement in decisions about their health. ${ }^{40}$ It consists of five 'cards' on a board, each illustrating a different role in decision-making by means of a cartoon and short descriptive statement. In its original version, administration requires a trained examiner, who asks the patient to choose the preferred card, which is then covered up. The procedure continues (four choices) until one card is left. If the second preference is incongruent with the first (non-adjacent pairing, such as card A with card C), the test is explained again, and immediately readministered. In the event of a further incongruence, the test is not readministered, and a preference is not assigned. Six scores are possible based on the subject's two most preferred roles: active-active, active-collaborative, collaborative-active, collaborativepassive, passive-collaborative and passive-passive. These scores are grouped as: active (active-active or activecollaborative), collaborative (collaborative-active or collaborative-passive) or passive (passive-collaborative or passive-passive).$^{40}$ We will use the electronic, Italian selfadministered CPS. ${ }^{42}$

\section{Quality of the conversation}

We will assess the quality of the first ACP conversation considering three perspectives: an independent observer, the pwPMS, and the physician. Each conversation will be unobtrusively audio-taped and transcribed verbatim; subsequently, a specially trained third observer will evaluate the behaviour of the physician in terms of patient involvement in decision-making using the OPTION (http://www.glynelwyn.com/observer-option-instrument.html). ${ }^{43}$ The OPTION consists of 12 items, each rated on a 5-point Likert scale ranging from 0 (behaviour not observed) to 4 (behaviour observed to high standard). A total score (range $0-48$ ) is obtained by adding the scores of each item. After the ACP conversation, pwPMS will complete the QOC $^{32}$; SOs will complete the SO version (QOC-SO), and physicians the physician version (last two items) of the QOC. Developed from qualitative studies with patients, families and clinicians, the QOC consists of 19 items measuring general communication (9 items) and communication about EOL care (8 items), each rated on a scale from 0 ('very worst I can imagine' / 'not at all') to 10 ('very best I can imagine' / 'extremely'), or identified as something the clinician did not do. The $0 / 10$ ratings are recoded to $1 / 11$, with 0 imputed for 'did not do' (http://depts.washington.edu/eolcare/ products/instruments/).

\section{Other outcome measures}

PwPMS quality of life will be assessed using the electronic version of the MSQOL-29, which is the shortened form of the MSQOL-54. ${ }^{38}$ MSQOL-29 includes 25 items forming 7 subscales and 4 single items, and 1 filter question for 3 'sexual function' items. Mood symptoms will be assessed with the HADS, a self-assessed questionnaire consisting of 14 multiple-choice (0-3 Likert scale) items probing symptoms of anxiety ( 7 items) and depression ( 7 items). HADS anxiety (HADS-A) and depression (HADS-D) scores range from 0 (no symptoms) to 21 (most severe symptoms). ${ }^{44}$ A cut-off score of 8 or above was recommended for patients with MS, since it was found to be an accurate indicator of major depression (90\% sensitivity, $87 \%$ specificity) and generalised anxiety disorder $\left(88.5 \%\right.$ sensitivity; $81 \%$ specificity) in this population. ${ }^{45}$ Finally, SO burden will be assessed using the ZBI, ${ }^{46}$ a 22-item self-report measure of subjective burden among caregivers addressing functional and behavioural impairments as well as the home care circumstances. A total 0 (low burden) to 88 (high burden) score is obtained by summing item responses, each scored on a 5-point Likert scale ranging from 0 (never) to 4 (nearly always present).

\section{Meetings}

There will be two study meetings (teleconferences): the investigators' meeting will be held before enrolment starts. Participants will be the Steering Committee, the centre PIs and the HPs who participated in the training programme. The aim of this meeting is to provide clear information on the study procedures, and to train HPs on the use of the trial platform. A second meeting will be run about 2 months after enrolment starts, in order to monitor possible difficulties, top-up centres' motivation and provide a safe place for peer discussion on the implementation of the intervention. Both meetings will last about 2 hours. Additional meetings will be organised whenever needed. In addition, the study PIs and the Training Panel will be available for inquiries about the implementation of the intervention at the participating centres.

\section{Nested qualitative study}

We will perform one-on-one semi-structured interviews with pwPMS and SOs, chosen using a maximum variation strategy, and FGMs of HPs involved in intervention delivery. For pwPMS and SOs interviews were considered most appropriate to limit interview burden and hopefully make it easier for participants to express their feelings, and recount their experiences of the intervention. For the patient referring physicians and the other HPs we chose FGMs as they promote interaction and exchange of ideas. A minimum of 10 interviews (five with pwPMS and five with SOs) and two FGMs will be held, the final number depending on the achievement of 'data saturation'. ${ }^{47}$ Interviews and FGMs will be run via video teleconference, which will ease participation of pwPMS with severe disability and SOs with caregiving commitments, as 
well as HPs. If the pwPMS and/or SOs have no access to internet using personal computer or other devices, such participants will be interviewed on the telephone.

The interviews aim to provide important feedback on participant perception of the quality of the intervention provided, and will serve as a process measure. Insights from this qualitative analysis will serve to inform finegrain intervention refinement. They will take place within 2 months of trial completion, and last no more than an hour. To reduce social desirability response bias, the interviewers will be researchers not involved in the ConCure-SM intervention delivery. Before starting, interviewees will be informed of study aims and requirements, and provide written consent. The interviewer will then explain that the aim of the interview is to obtain participant feedback on experience of the pilot study and stress that positive and negative experiences of, and feelings about, the intervention are welcome. Participants will be assured that the interviews are confidential, and that the audio recordings and subsequent transcripts will be fully anonymised. The interviewer will then pose each question in turn, neutrally (so as to not suggest any particular reply) and in an open-ended fashion (to allow many possible replies). As each question is discussed, follow-up questions will clarify and explore participant responses. Participants will be also encouraged to elaborate on any pertinent themes or views that emerge. The interviewer will note any potentially informative non-verbal gestures. At the end of the interview, the interviewer will verbally summarise the key points and ask the participant if the summary is full and correct.

The FGMs aim to collect insights and living experiences about the intervention and to identify possible barriers to its implementation; they will provide important feedback on the intervention and on factors that can enable its implementation and adoption. For this reason, HPs other than the physicians involved in the ACP conversation will be involved. Each FGM (teleconference) will last about 2 hours; participants will be $6-10$ physicians who delivered the intervention and HPs from the participating centres. All participants will provide written informed consent prior to the FGM, that will be conducted by two psychologists specifically trained in qualitative research. One will be the facilitator, whose job is to engage all participants, promote exchange, moderate conflicts, ensure that all prespecified topics will be adequately covered, and allow exploration of any pertinent issues that arise. He/she will first explain the purpose of the meeting and ask participants to introduce themselves. He/she will then introduce each topic in turn, in an open-ended fashion. At any point, the facilitator can probe for further information and ask follow-up questions to stimulate further discussion. After all prespecified topics are fully discussed, the facilitator will summarise the main points, and ask for further feedback and whether all concerns have been fully aired. The comoderator will take notes and oversee the audio recording. Subsequently, they will produce a report from the audio recordings/transcript and field notes, which will be submitted to participants for review (respondent validation).

\section{Data analysis}

Study power

As this is a pilot and feasibility study, a formal sample size calculation is not required. We aim to recruit at least 40 pwPMS from six centres to assess feasibility across a diverse range of participants including those with different care needs and living conditions. There are no data available on the occurrence of ACP in pwPMS: by hypothesising a proportion in the pwPMS population of $10 \%$, a sample size of 35 subjects achieves a power of $90 \%$, assuming a type I error of 5\%, to detect a proportion of ACP documentation of $30 \%$. By hypothesising a proportion in the pwPMS population of $8 \%$, a sample size of 35 subjects achieves a power of $95 \%$, assuming a type I error of $5 \%$, to detect a proportion of ACP documentation of $30 \%$. By adding $15 \%$ of drop outs or incomplete data, 40 pwPMS should be recruited.

\section{Statistics}

Descriptive statistics will be calculated for general and clinical variables. Specifically, continuous variables will be summarised by their mean and $\mathrm{SD}$, or median and IQR; categorical variables will be summarised as numbers and percentages. Categorical variables will be compared using the $\chi^{2}$ test. The normality assumption of continuous variables will be tested with the Shapiro-Wilk test. Depending on data distribution, between-group comparisons will be carried out using either the two-sided unpaired t-test or the Wilcoxon two-sided two sample test; within-group comparisons will be carried out using either the paired t-test or the Wilcoxon signed-rank test; correlations will be computed using Pearson's or Spearman's coefficients.

Our primary end-point is the proportion of pwPMS completing an ACP during the 6-month period. Change in the secondary outcome measures will be also calculated. In addition, we will calculate the following feasibility outcomes: recruitment rate (enrolment per month; reasons for non-eligibility, non-enrolment); retention rate (proportion completing the intervention and study follow-up); missing data (proportion fully completed, for each scale, at each time point). Data will be analysed according to the intention-to-treat principle. Multiple imputation of missing values will employ Rubin's approach. A p value less than 0.05 will be considered statistically significant. No correction for multiple comparisons will be applied. All analyses will be performed using STATA V.16. Assumptions in determining the sample size of the main trial will be checked.

\section{Qualitative data}

Interviews and FGMs will be audio-recorded and transcribed verbatim. Data analysis will be conducted by three researchers with experience in qualitative research. Researchers will analyse interviews and FGM data using thematic analysis, with interpretation guided by the four 
normalisation process theory (NPT) components (see process evaluation below). Data will be triangulated across sources. The analytical stages can be summarised as follows ${ }^{48}$ : (1) each researcher will read the transcriptions and write comments and initial thoughts in a memo. (2) Each researcher will extract portions of the text individually and then share their work to reach an initial agreement. During this stage, they will conduct the thematic analysis inductively providing their insights. (3) Researchers will independently review themes and allocate portions of the text to the newly reconfigured themes. (4) Together, they will redefine themes and rename them to achieve internal consistency. (5) One researcher will extract from the interviews and draft the final report, which will be checked and amended by the other two.

\section{Process evaluation}

We will follow the Medical Research Council guidance on process evaluation, ${ }^{49}$ which describes three components using a mixed-methods approach: implementation or delivery; mechanisms of impact; contextual factors. We will use NPT to determine if, and in what ways, the ConCure-SM intervention can be successfully 'normalised' (embedded) into clinical practice.$^{50}{ }^{51}$ At the feasibility and piloting stage, basic quantitative measures of implementation may be combined with in-depth qualitative data to provide detailed understandings of intervention functioning on a small scale. ${ }^{49}$ Quantitative measures will include structured observations of audio-recorded ACP conversations. These will be used to examine aspects of fidelity (such as consistency with the Shared Decision Making principles), and dose (the duration of conversations). Qualitative methods will be used to investigate mechanisms of impact and contextual factors, using NPT. NPT identifies four essential determinants of 'normalising' complex interventions into common practice: coherence (the extent to which an intervention is understood as being meaningful, achievable and valuable); cognitive participation (the engagement of HPs necessary to deliver the intervention); collective action (the work that brings the intervention into use) and reflexive monitoring (the on-going process of adjusting the intervention to keep it in place).$^{51}$ These components are considered to be dynamic and interact within the wider context of the intervention, such as existing organisational structures and procedures. ${ }^{51}$ Further, we will use qualitative data to identify required modifications and to develop practical strategies for enabling and sustaining intervention delivery in clinical settings.

\section{Patient and public involvement statement}

An expert patient with MS and a representative of the Italian MS Society are part of the Steering Committee of the project and coauthors of the present paper. These same persons were part of the interdisciplinary panel that produced the ACP booklet, which was revised based on the results of a qualitative study with users (pwPMS, SOs and HPs).

Prior to designing and conducting a full trial, the intervention will be pilot tested in a multicentre study involving MS and rehabilitation centres across Italy, and using a mixed-method approach.

We will disseminate key study findings to pwPMS via the Italian MS Society.

\section{Ethics and dissemination}

The project is coleaded by a neurologist and a bioethicist. Phase 1 has received ethical approvals from each participating centre, while phase 2 will be submitted to the centres in May 2021. Findings from both phases will be disseminated widely through peer-reviewed publications, conferences and workshops. Authorship eligibility will be based on The International Committee of Medical Journal Editors. The final trial (pseudo-anonymised) dataset will be accessed by the study PIs and the data management/analysis team. Details about panels and centres, ethics and administrative considerations, and study management and monitoring are available in online supplemental appendix 3.

\section{DISCUSSION}

One of the 10 clinical questions of the European Academy of Neurology (EAN) guideline on palliative care of pwPMS specifically addressed ACP. ${ }^{17}$ For this clinical question, formulated with direct patient and caregiver involvement, ${ }^{52}$ no evidence was found and two good practice statements were produced: 'It is suggested that early discussion of the future with ACP is offered to patients with severe MS'; 'It is suggested that regular communication about the future progression of MS is undertaken with patients and families/caregivers' ${ }^{17}$ To fill this knowledge gap, we conceived the present study, which adheres to the Shared Decision Making model, ${ }^{12-14}$ and to the Medical Research Council framework for developing and evaluating complex interventions. ${ }^{24}$ Within this methodological context, the study follows the CONSORT guidance for trials on social and psychological interventions (CONSORT-SPI 2018), ${ }^{27}$ as many of the guidance items (excluding items that are specific to the randomisation nature of the study) are relevant for reporting other types of pilot and feasibility studies. ${ }^{53}$ This includes the development of the study protocol following the SPIRIT guidance ${ }^{26}$ protocol's publication and the trial public registration (ISRCTN registry). The consolidated criteria for reporting qualitative research will guide the presentation of findings in the study reports and publications. ${ }^{2854}$

To increase generalisability of the study, participants (pwPMS, SOs and HPs) will be enrolled from university hospitals, research hospitals and clinical centres from the different areas of Italy. We will not enrol from primary care practices as in Italy patients with MS are followed in tertiary care centres. Moreover, previous attempts to 
involve the family physicians in the care of PwPMS were challenging. ${ }^{55}$

Personal, semi-structured interviews and FGMs will be run via video teleconference, which will ease participation of pwPMS with severe disability and SOs with caregiving commitments, as well as HPs. If pwPMS and/or SOs have no internet access, using personal computer or other devices, these participants will be interviewed on the telephone. Other measures adopted to minimise bias include: all study personnel will be trained to conform to GCP regulation; electronic version of the study questionnaires/inventories will be used to ensure the data entered is of high quality; an DSMC will monitor and supervise the progress of the trial, and the safety data.

The ConCure-SM intervention (booklet and HP training programme) can be adapted for use in other neurological and non-neurological conditions for which consolidated ACP interventions are not available. The electronic format will ease the incorporation of the advance care plan document (and its updates) in the electronic medical record, that is currently available in some Italian regions and hopefully will be soon available all over Italy.

\section{Study limitations}

Three study limitations are noted. We used a single-arm design for the pilot trial. This decision was taken as ACP is currently at premium in MS, ${ }^{17} 23$ and designing a randomised (cluster) trial with standard care or any 'low intensity' intervention as a comparator was considered ethically and practically unviable. Another limitation is that our training programme was for HPs only. A multiple-component intervention that targets clinicians and patients simultaneously has been suggested in other disciplines. ${ }^{56}$ In the current situation regarding ACP, we preferred to have a clear focus on enhancing HP competencies. ${ }^{1723}$ Finally, our pilot trial lacks long-term outcomes, chiefly the concordance between preferred and received EOL care and treatments. ${ }^{57}$ However, the MS trajectory further challenges the collection of this outcome in the typical timeframe of a clinical trial. In line with the principles of ACP, we agreed not to narrow the inclusion criteria only to pwPMS in the late stage of the disease, deserving this relevant outcome to future studies.

\footnotetext{
Author affiliations

${ }^{1}$ Bioethics Unit, Azienda USL - IRCCS di Reggio Emilia, Reggio Emilia, Italy

${ }^{2}$ Fondazione FARO, Turin, Italy

${ }^{3}$ The Italian Multiple Sclerosis Society, Genoa, Italy

${ }^{4}$ Hospice "La Torre sul Colle", USL Umbria 2, Spoleto (PG), Italy

${ }^{5}$ Department of Neuroscience, Biomedicine, and Movement Sciences, University of

Verona, Verona, Italy

${ }^{6}$ Unit of Neurology, Borgo Roma Hospital, Azienda Ospedaliera Universitaria Integrata Verona, Verona, Italy

${ }^{7}$ Multiple Sclerosis Unit, IRCCS S. Lucia Foundation, Rome, Italy

${ }^{8}$ EUPATI Fellow (European Patients Academy for Therapeutic Innovation) Italy, Roma, Italy

${ }^{9}$ UOSI Riabilitazione Sclerosi Multipla, IRCCS Istituto delle Scienze Neurologiche di Bologna, Bologna, Italy
}

${ }^{10}$ Dipartimento di Scienze Biomediche e Neuromotorie, Università di Bologna, Bologna, Italy

${ }^{11}$ New Zealand Health Quality and Safety Commission, Nelson, New Zealand

${ }^{12}$ Multiple Sclerosis Center, Azienda USL-IRCCS di Reggio Emilia, Reggio Emilia, Italy

${ }^{13}$ Multiple Sclerosis Center, University Hospital Policlinico Vittorio Emanuele, Catania, Italy

${ }^{14}$ UOC Neurologia, ASUR Marche, Fermo, Italy

${ }^{15}$ Department of Rehabilitation, M.L. Novarese Hospital, Moncrivello (VC), Italy

${ }^{16}$ Unit of Neuroepidemiology, Fondazione IRCCS Istituto Neurologico Carlo Besta,

Milano, Italy

Acknowledgements We are indebted with the pwPMS, SOs and the HPs who cognitively debriefed the provisional version of the booklet, with Kasia Nowak and Andrea Vitali (booklet layout), Chiara Uncini and Nicola Lugaresi (images). We thank the 'Associazione Marchigiana Sclerosi Multipla ed altre Malattie Neurologiche' for supporting the production of the provisional version of the booklet.

Collaborators ConCure-SM Steering Committee: LDP, SV, MB, MC, MGG, PK, AL, SM, FP, EP, CS, AGiordano, AS. Data Safety and Monitoring Committee: Kevin Brazil, School of Nursing and Midwifery, Queen's University of Belfast, Belfast, Northern Ireland, UK; Bobbie Farsides, Brighton; Luciano Orsi, The Italian Society of Palliative Care (SICP), Milan, Italy; Carlo Peruselli, SICP, Milan, Italy; and David Oliver, The Tizard Centre, University of Kent, Canterbury, UK (Chair). Data Management and Analysis Committee: AGiordano, Mariangela Farinotti, Unit of Neuroepidemiology, Fondazione IRCCS Istituto Neurologico Carlo Besta, Milan, Italy. Qualitative Analysis Panel: LDP, SV, MC, Luca Ghirotto, Qualitative Research Unit, Azienda USL-IRCCS di Reggio Emilia, Reggio Emilia, Italy; Katia Mattarozzi, Department of Experimental, Diagnostic and Specialistic Medicine, School of Medicine, Alma Mater Studiorum University of Bologna, Italy; Marta Perin, Unit of Bioethics, Azienda USL-IRCCS di Reggio Emilia, Reggio Emilia, Italy. HP Training Panel: LDP, SV, MC, KM, EP, Michela Rimondini, Section of Clinical Psychology, Department of Neuroscience, Biomedicine and Movement Sciences, University of Verona, Policlinico G.B. Rossi, Verona, Italy; AS. Linguistic Validation Panel: MF, PK, SV, AGiordano, AS. Enrolling Centers and Investigators: Department of Neuroscience, Biomedicine and Movement Sciences, University of Verona; Unit of Neurology, Borgo Roma Hospital, Azienda Ospedaliera Universitaria Integrata Verona, Verona, Italy: AGajofatto, Francesca Gobbin, Riccardo Orlandi. Department of Rehabilitation M. L. Novarese Hospital, Moncrivello, Vercelli: CS, Enrica Grange. Multiple Sclerosis Center, Azienda USL-IRCCS di Reggio Emilia, Reggio Emilia, Italy: SM, Francesca Sireci. UOSI Riabilitazione Sclerosi Multipla, IRCCS Istituto delle Scienze Neurologiche di Bologna; Dipartimento di Scienze Biomediche e Neuromotorie, Università di Bologna, Bologna: AL, Loredana Sabbatini, Cinzia Scandellari, Elisa Ferriani. Fondazione IRCCS Santa Lucia, Roma: MGG, Giorgia Presicce. University Hospital Policlinico Vittorio Emanuele, Catania: FP, Clara Grazia Chisari, Simona Toscano.

Contributors LDP, SV and AS conceived and developed the study protocol. LDP, SV MB, MC, AGajofatto, MGG, PK, AL, LM, SM, FP, EP, CS, AGiordano and AS contributed to the refinement of the study protocol. LDP, SV and AS drafted the manuscript. LDP, SV, MB, MC, AGajofatto, MGG, PK, AL, LM, SM, FP, EP, CS, AGiordano and AS approved the final manuscript.

Funding Phase 2 was supported by Fondazione Italiana Sclerosi Multipla (FISM; aism.fism.it), grant no. 2020/R-Multi/024 to AS. The funding source had no role in study design, data collection, data analysis, data interpretation or report writing.

Competing interests AL reports grants from Novartis, during the conduct of the study; personal fees from Biogen, Merck Serono, Mylan, Novartis, Roche, Sanofi/ Genzyme, Teva and FISM. FP received personal compensation for serving on advisory board and/or speaking activities by Almirall, Bayer, Biogen, Bristol Meyers \& Squibb, Merck, Novartis Roche, Sanofi and TEVA; he further received research grants by Biogen Italy, Biogen Global, Merck, University of Catania, FISM and Reload Onlus Patients Association. AS reports grants from FISM and European Academy of Neurology, during the conduct of the study; personal fees from Almirall and Merck Serono. This does not alter our adherence to BMJ Open policies on sharing data. All the other authors report no competing interests.

Patient consent for publication Obtained.

Provenance and peer review Not commissioned; externally peer reviewed.

Supplemental material This content has been supplied by the author(s). It has not been vetted by BMJ Publishing Group Limited (BMJ) and may not have been peer-reviewed. Any opinions or recommendations discussed are solely those of the author(s) and are not endorsed by BMJ. BMJ disclaims all liability and responsibility arising from any reliance placed on the content. Where the content 
includes any translated material, BMJ does not warrant the accuracy and reliability of the translations (including but not limited to local regulations, clinical guidelines, terminology, drug names and drug dosages), and is not responsible for any error and/or omissions arising from translation and adaptation or otherwise.

Open access This is an open access article distributed in accordance with the Creative Commons Attribution Non Commercial (CC BY-NC 4.0) license, which permits others to distribute, remix, adapt, build upon this work non-commercially, and license their derivative works on different terms, provided the original work is properly cited, appropriate credit is given, any changes made indicated, and the use is non-commercial. See: http://creativecommons.org/licenses/by-nc/4.0/.

\section{ORCID iD}

Alessandra Solari http://orcid.org/0000-0001-9930-7579

\section{REFERENCES}

1 Browne P, Chandraratna D, Angood C, et al. Atlas of multiple sclerosis 2013: a growing global problem with widespread inequity. Neurology 2014;83:1022-4

2 Filippi M, Bar-Or A, Piehl F, et al. Multiple sclerosis. Nat Rev Dis Primers 2018:4:43.

3 Kingwell E, van der Kop M, Zhao Y, et al. Relative mortality and survival in multiple sclerosis: findings from British Columbia, Canada. J Neurol Neurosurg Psychiatry 2012;83:61-6.

4 Scalfari A, Knappertz V, Cutter G, et al. Mortality in patients with multiple sclerosis. Neurology 2013;81:184-92.

5 Lunde HMB, Assmus J, Myhr K-M, et al. Survival and cause of death in multiple sclerosis: a 60-year longitudinal population study. J Neurol Neurosurg Psychiatry 2017;88:621-5.

6 Chiaravalloti ND, DeLuca J. Cognitive impairment in multiple sclerosis. Lancet Neurol 2008;7:1139-51.

7 Higginson IJ, Hart S, Silber E, et al. Symptom prevalence and severity in people severely affected by multiple sclerosis. J Palliat Care 2006;22:158-65

8 Hirst C, Swingler R, Compston DAS, et al. Survival and cause of death in multiple sclerosis: a prospective population-based study. $J$ Neurol Neurosurg Psychiatry 2008;79:1016-21.

9 Sumelahti M-L, Hakama M, Elovaara I, et al. Causes of death among patients with multiple sclerosis. Mult Scler 2010;16:1437-42.

10 Giordano A, Ferrari G, Radice D, et al. Health-related quality of life and depressive symptoms in significant others of people with multiple sclerosis: a community study. Eur J Neurol 2012;19:847-54.

11 Rietjens JAC, Sudore RL, Connolly M, et al. Definition and recommendations for advance care planning: an international consensus supported by the European association for palliative care. Lancet Oncol 2017;18:e543-51.

12 Archer J, Stevenson L, Coulter A, et al. Connecting patient experience, leadership, and the importance of involvement, information, and empathy in the care process. Healthc Manage Forum 2018;31:252-5.

13 Coulter A, Collins A. Making shared decision-making a reality. London, United Kingdom: King's Fund, 2011. https://www.kingsfund. org.uk/publications/making-shared-decision-making-reality

14 Elwyn G, Frosch D, Thomson R, et al. Shared decision making: a model for clinical practice. J Gen Intern Med 2012;27:1361-7.

15 Forte DN, Kawai F, Cohen C. A bioethical framework to guide the decision-making process in the care of seriously ill patients. BMC Med Ethics 2018;19:78.

16 De Panfilis L, Giorgi Rossi P, Mazzini E, et al. Knowledge, opinion and attitude about the Italian law on advance directives: a populationbased survey. J Pain Symptom Manage 2020;S0885-3924:30561-3.

17 Solari A, Giordano A, Sastre-Garriga J, et al. EAN guideline on palliative care of people with severe, progressive multiple sclerosis. Eur J Neurol 2020;27:1510-29.

18 Brinkman-Stoppelenburg A, Rietjens JAC, van der Heide A. The effects of advance care planning on end-of-life care: a systematic review. Palliat Med 2014;28:1000-25.

19 Golla H, Galushko M, Strupp J, et al. Patients feeling severely affected by multiple sclerosis: addressing death and dying. Journal of Death \& Dying 2016;74:275-91.

20 Golla H, Mammeas S, Galushko M, et al. Unmet needs of caregivers of severely affected multiple sclerosis patients: a qualitative study. Palliat Support Care 2015;13:1685-93.

21 Walter HAW, Seeber AA, Willems DL, et al. The role of palliative care in chronic progressive neurological diseases-a survey amongst neurologists in the Netherlands. Front Neurol 2018;9:1157.

22 McCurry MK. An exploratory study of decision making by informal caregivers of individuals with multiple sclerosis. J Neurosci Nurs 2013;45:52-60.
23 Cottrell L, Economos G, Evans C, et al. A realist review of advance care planning for people with multiple sclerosis and their families. PLoS One 2020;15:e0240815.

24 Craig P, Dieppe P, Macintyre S, et al. Developing and evaluating complex interventions: the new medical Research Council guidance. BMJ 2008;337:a1655.

25 Chan A-W, Tetzlaff JM, Gøtzsche PC, et al. SPIRIT 2013 explanation and elaboration: guidance for protocols of clinical trials. BMJ 2013;346:e7586.

26 Calvert M, Kyte D, Mercieca-Bebber R, et al. Guidelines for inclusion of patient-reported outcomes in clinical trial protocols: the SPIRITPRO extension. JAMA 2018;319:483-94.

27 Grant S, Mayo-Wilson E, Montgomery P, et al. CONSORT-SPI 2018 explanation and elaboration: guidance for reporting social and psychological intervention trials. Trials 2018;19:406.

28 Tong A, Sainsbury P, Craig J. Consolidated criteria for reporting qualitative research (COREQ): a 32-item checklist for interviews and focus groups. Int J Qual Health Care 2007;19:349-57.

29 Guillemin F, Bombardier C, Beaton D. Cross-cultural adaptation of health-related quality of life measures: literature review and proposed guidelines. J Clin Epidemiol 1993;46:1417-32.

30 Wild D, Eremenco S, Mear I, et al. Multinational trialsrecommendations on the translations required, approaches to using the same language in different countries, and the approaches to support pooling the data: the ISPOR patient-reported outcomes translation and linguistic validation good research practices Task force report. Value Health 2009;12:430-40.

31 Sudore RL, Heyland DK, Barnes DE, et al. Measuring advance care planning: optimizing the advance care planning engagement survey. $J$ Pain Symptom Manage 2017;53:669-81.

32 Engelberg R, Downey L, Curtis JR. Psychometric characteristics of a quality of communication questionnaire assessing communication about end-of-life care. J Palliat Med 2006;9:1086-98.

33 Lublin FD, Reingold SC, National Multiple Sclerosis Society (USA) Advisory Committee on Clinical Trials of New Agents in Multiple Sclerosis*. Defining the clinical course of multiple sclerosis: results of an international survey. Neurology 1996;46:907-11.

34 Downar J, Goldman R, Pinto R, et al. The "surprise question" for predicting death in seriously ill patients: a systematic review and meta-analysis. CMAJ 2017;189:E484-93.

35 Giordano A, Mattarozzi K, Pucci E, et al. Participation in medical decision-making: attitudes of Italians with multiple sclerosis. J Neurol Sci 2008;275:86-91.

36 Costantini M, Musso M, Viterbori P, et al. Detecting psychological distress in cancer patients: validity of the Italian version of the hospital anxiety and depression scale. Support Care Cancer 1999;7:121-7.

37 Goss C, Fontanesi S, Mazzi MA, et al. [The assessment of patient involvement across consultation. The Italian version of the OPTION scale]. Epidemiol Psichiatr Soc 2007;16:339-49.

38 Rosato R, Testa S, Bertolotto A, et al. eMSQOL-29: prospective validation of the abbreviated, electronic version of MSQOL-54. Mult Scler 2019;25:856-66.

39 Chattat R, Cortesi V, Izzicupo F, et al. The Italian version of the Zarit burden interview: a validation study. Int Psychogeriatr 2011;23:1-9.

40 Degner LF, Sloan JA, Venkatesh P. The control preferences scale. Can J Nurs Res 1997;29:21-43.

41 Kryworuchko J, Stacey D, Bennett C, et al. Appraisal of primary outcome measures used in trials of patient decision support. Patient Educ Couns 2008;73:497-503.

42 Solari A, Giordano A, Kasper J, et al. Role preferences of people with multiple sclerosis: image-revised, computerized selfadministered version of the control preference scale. PLoS One 2013;8:e66127.

43 Elwyn G, Hutchings $\mathrm{H}$, Edwards A, et al. The OPTION scale: measuring the extent that clinicians involve patients in decisionmaking tasks. Health Expect 2005;8:34-42.

44 Zigmond AS, Snaith RP. The hospital anxiety and depression scale. Acta Psychiatr Scand 1983;67:361-70.

45 Honarmand K, Feinstein A. Validation of the hospital anxiety and depression scale for use with multiple sclerosis patients. Mult Scler 2009;15:1518-24.

46 Hébert R, Bravo G, Préville M. Reliability, validity and reference values of the Zarit burden interview for assessing informal caregivers of community-dwelling older persons with dementia. Can J Aging 2000;19:494-507.

47 Denzin NK, Lincoln YS. Handbook of qualitative research. London, UK: Sage Publications, 2000.

48 Braun V, Clarke V. Using thematic analysis in psychology. Qual Res Psychol 2006;3:77-101. 
49 Moore G, Audrey S, Barker M. Process evaluation of complex interventions: medical Research Council guidance. 7. London: MRC Population Health Science Research Network, 2014.

50 Murray E, Treweek S, Pope C, et al. Normalisation process theory: a framework for developing, evaluating and implementing complex interventions. BMC Med 2010;8:63.

51 May CR, Cummings A, Girling M, et al. Using normalization process theory in feasibility studies and process evaluations of complex healthcare interventions: a systematic review. Implement Sci 2018;13:80.

52 Köpke S, Giordano A, Veronese S, et al. Patient and caregiver involvement in the formulation of guideline questions: findings from the European Academy of Neurology guideline on palliative care of people with severe multiple sclerosis. Eur J Neurol 2019;26:41-50.

53 Lancaster GA, Thabane L. Guidelines for reporting non-randomised pilot and feasibility studies. Pilot Feasibility Stud 2019;5:114.
54 O'Brien BC, Harris IB, Beckman TJ, et al. Standards for reporting qualitative research: a synthesis of recommendations. Acad Med 2014;89:1245-51.

55 Giovannetti AM, Borreani C, Bianchi E, et al. Participant perspectives of a home-based palliative approach for people with severe multiple sclerosis: a qualitative study. PLoS One 2018;13:e0200532.

56 Schichtel M, Wee B, Perera R, et al. Clinician-targeted interventions to improve advance care planning in heart failure: a systematic review and meta-analysis. Heart 2019;105:1316-24.

57 Higginson IJ, Evans CJ, Grande G, et al. Evaluating complex interventions in end of life care: the MORECare statement on good practice generated by a synthesis of transparent expert consultations and systematic reviews. BMC Med 2013;11:111.

58 Kurtzke JF. Rating neurologic impairment in multiple sclerosis: an expanded disability status scale (EDSS). Neurology 1983;33:1444-52.

59 Mahoney FI, Barthel DW. Functional evaluation: the BARTHEL index. Md State Med J 1965;14:56-61. 